03

\title{
Управляемое электрическим полем передвижение жидких капель по поверхности диэлектрических пленок
}

\author{
() А.М. Орлов, М.Ю. Махмуд-Ахунов, К.В. Кузнецова
}

Ульяновский государственный университет, 432000 Ульяновск, Россия

e-mail: maratmau@mail.ru

(Поступило в Редакцию 19 июня 2017 г. В окончательной редакции 27 апреля 2018 г.)

\begin{abstract}
Представлены результаты исследований кинетики перемещения капель воды по поверхности экранированных металлических электродов под действием внешнего электрического поля. Установлено, что определяющую роль в механизме перемещения играет разница краевых углов смачивания отдельных участков капли, расположенной на управляемых электродах. Показано, что перемещение капли независимо от полярности управляющих электродов и заземления для рассматриваемой системы диэлектриков всегда происходит в направлении отрицательного электрода. Теоретический анализ представленных результатов проведен на основании уравнения Липпмана, переписанного для поляризованной капли, локализованной на двух управляющих электродах, экранированных многослойным диэлектриком.
\end{abstract}

DOI: 10.21883/JTF.2018.11.46622.2389

Управляемое током изменение геометрии жидких капель и их перемещение по твердой поверхности открывает практическую перспективу использования таких капель в качестве основных рабочих элементов микродозаторов [1], уголковых [2,3] и пластинчатых [4] отражателей, микролинз с управляемым фокусным расстоянием [5], цифровых дисплеев [6]. Управляющим фактором геометрических изменений в поляризованной капле является перестройка двойного электрического слоя (double-electric layer - DEL) контактной зоны, в которой под действием прикладываемого напряжения $U$ меняется поверхностный заряд $q_{S}\left(\mathrm{C} / \mathrm{m}^{2}\right)$, что в соответствии с термодинамическим уравнением Липпмана [7]

$$
\frac{d \sigma}{d U}=-q_{S}
$$

приводит к изменению поверхностного натяжения $\sigma$ жидкости $(L)$, контактирующей с твердой $(S)$ подложкой: $\sigma=\sigma_{L S}, \mathrm{~J} / \mathrm{m}^{2}$.

Эти изменения через краевой угол смачивания $\theta$ [8] оказывают существенное влияние на растекание жидкости по подложке. Простота трансформации $\theta$ электрическим полем активизирует и расширяет практическую область применения столь простого способа управляемой смачиваемости. Так, ассиметричное деформирование капли, расположенной на двух экранированных электродах, обеспечивает значительные различия краевых углов смачивания отдельных участков одной и той же капли, приводящее к ее смещению или полному переходу с одной площадки на другую. В результате капля, располагаясь на экранированной многоэлектродной матрице, может быть доставлена электрическим полем в любую ее точку по заранее заданному маршруту [1].

К сожалению, теоретические аспекты управляемого перемещения капель по многоэлектродной матрице далеки от завершения и нуждаются в дальнейшей проработке. Анализ подобных явлений обычно основывается на теории Гельмгольца-Смолуховского [9], на эмпирических или полуэмпирических уравнениях, описывающих эксперимент [10], либо на классическом уравнении Липпмана [8] для поляризованной в ионном растворе металлической капли или же ограничивается рассмотрением системы с верхним электродом (проволочным или пластинчатым) и нижней управляющей матрицей разделенных электродов [11-13]. Однако нами не было встречено работ по описанию динамики перемещения поляризованной капли с позиций электроемкостных характеристик отдельных ее фрагментов, располагавшихся на двух электродах многоэлектродной матрицы, управляющих численными значениями $q_{S}$ и $\sigma_{L S}$ контактных зон. В сочетании с электрокапиллярной кривой такой подход позволяет объяснить не только кинетику перемещения поляризованной капли (направления и скорости), но и вскрыть причины инверсионного ее перемещения при нарастающем потенциале той же полярности. Учитывая это, попытаемся вскрыть особенности поведения капли в эффективном поле двух поляризованных электродов, экранированных двуслойным диэлектриком.

В проводимых нами исследованиях капля дистиллированной воды помещалась на одну из экранированных площадок многоэлектродной матрицы. Изолированные между собою электроды имели форму квадрата площадью $\sim 3 \times 3 \mathrm{~mm}^{2}$ с пилообразными взаимно проникающими выступами. Контактный размер капель приблизительно соответствовал стороне квадрата, описывающего наружный контур медного электрода. Контактные площадки могут быть и прямоугольными. Важно, чтобы, полностью располагаясь на одном электроде, капля своими периферийными участками касалась соседних электродов. Только в этом случае можно обеспечить непрерывность ее перемещения в любом из заданных направлений, хотя более плавный переход с одной 
площадки на другую реализуется при пилообразных взаимно проникающих выступах.

Многоэлектродная матрица изготавливалась из стеклотекстолита с двухсторонней металлизацией $(\mathrm{Cu})$. На одной из сторон вытравливалась сетка межэлектродных зазоров шириной $0.4 \mathrm{~mm}$, на другой формировалась разводка к электродным площадкам первого слоя. Электрический контакт соответствующих площадок с металлизированными дорожками второго слоя обеспечивался стандартной пайкой соединительных перемычек $\varnothing 0.7 \mathrm{~mm}$ с последующей планарной шлифовкой и механической полировкой рабочей поверхности пластины.

Вся поверхность пластины экранировалась. Первый экранирующий слой электродной поверхности представлен полиэтиленовой пленкой толщиной $\sim 8 \mu \mathrm{m}$, обладающей хорошими диэлектрическими свойствами, но повышенной гидрофильностью. Для достижения оптимальной смачиваемости наносился второй экранирующий слой в виде раствора тефлона AF C5-18, обладающий после сушки худшими диэлектрическими, но лучшими гидрофобными свойствами. Скольжение полиэтиленовой пленки по медному электроду предотвращалось их склеиванием тончайшим слоем силиконового масла (ПМС-5). Внешнее питание к паре активных электродов подавалось от источника постоянного тока Б5-47 либо от заряженного до необходимого напряжения $(100-300 \mathrm{~V})$ блока параллельно соединенных конденсаторов (К75-40a, $3000 \mathrm{~V}, 100 \mu \mathrm{F})$ общей емкостью $300 \mu \mathrm{F}$. Последовательное переключение рабочих электродов, обеспечивающих перемещение капли на вновь подключенные площадки, осуществлялось специальным электронным блоком, управляемым компьютером. В случае одного поляризованного электрода, предназначенного для индуцирования электрических зарядов на межфазных границах капли, использовался высоковольтный источник с регулируемым $(0-15 \mathrm{kV})$ выходным напряжением. Измерение краевых углов смачивания осуществлялось с помощью оптического микроскопа МБС-10, сопряженного с цифровой камерой Levenhuk C35.

Проведенные нами опыты показали уверенное перемещение капли по многоэлектродной матрице по любому заранее запрограммированному маршруту. Скорость ее перемещения менялась в диапазоне $\sim(2-112) \mathrm{mm} / \mathrm{s}$ и с увеличением напряжения возрастала по экспоненциальному закону (рис. 1). Проанализируем природу перемещения капли с одного электрода на другой.

На первом экспериментальном этапе мы выяснили характер изменения электрической емкости $C$ неполяризованной капли, механически смещая ее с одной площадки на другую. Для этого чувствительный мультиметр подключался к контактным площадкам, а смещение капли осуществлялось вручную диэлектрической палочкой $\varnothing 0.8 \mathrm{~mm}$, погруженной в наивысшую точку капли на глубину $0.2-0.4 \mathrm{~mm}$. Эти результаты представлены на рис. 1. Видно, что наименьшим значениям емкости соответствуют стартовые и финишные позиции, когда

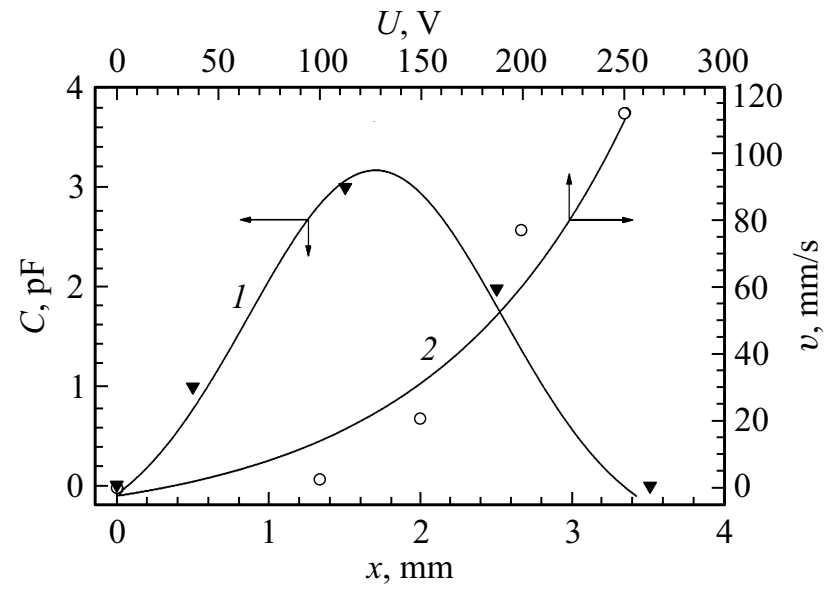

Рис. 1. Изменение результирующей емкости $C(x)$ неполяризованной капли в процессе ее механического смещения с одной электродной площадки на другую (нуль соответствует стартовому расположению всей капли на одной из площадок) (1) и влияние напряжения на скорость перемещения капли (2).
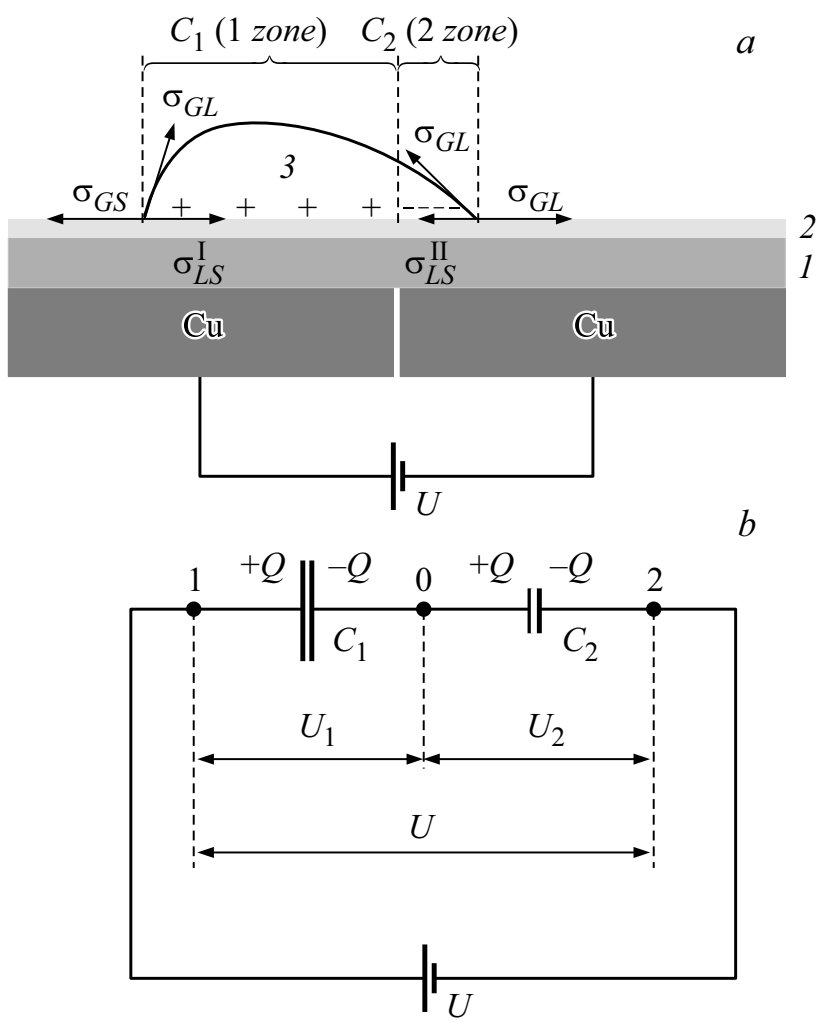

Рис. 2. Поляризованная капля жидкости на диэлектрической пленке $(a)$ и эквивалентная схема $(b)$, представленная двумя результирующими конденсаторами. Плоскость стыковки двух конденсаторов $(a)$ неподвижна и всегда располагается над линией, разделяющей медные электроды. На рис. $a$ : 1 полиэтиленовая пленка; 2 - тефлон; 3 - дистиллированная $\mathrm{H}_{2} \mathrm{O}$.

капля полностью располагается над одним из электродов. Наибольшему значению $C$ отвечает приблизитель- 
ное равенство площадей двух электродных площадок, накрываемых каплей. Следовательно, фиксируемое опытом экстремальное изменение результирующей емкости столь сложной системы может быть представлено двумя последовательно соединенными переменными конденсаторами $C_{1}$ и $C_{2}$ (рис. 2)

$$
C=\frac{C_{1} \cdot C_{2}}{C_{1}+C_{2}}
$$

емкости которых синхронно и взаимосвязано меняются в процессе перемещения капли с одной электродной площадки на другую. Наружные обкладки этих конденсаторов представлены медным электродом и диполями молекул $\mathrm{H}_{2} \mathrm{O}$, ориентированными вдоль поля в стыкующейся плоскости этих конденсаторов (рис. 2,a). Так что пространство между наружными обкладки рассматриваемых конденсаторов заполнено трехслойным диэлектриком, представленным полиэтиленом, тефлоном и соответствующим фрагментом капли дистиллированной $\mathrm{H}_{2} \mathrm{O}$.

Численное значение емкости $C$ в экстремальной точке $\left(C=C^{*}\right)$ рис. 1 оказалось равным $\sim 3.2 \mathrm{pF}$, что позволило определиться с параметрами сопряженных конденсаторов $C_{1}^{*}=C_{2}^{*}=2 C^{*} \approx 6.4 \mathrm{pF}(2)$ на этом участке кривой и с диапазоном синхронного изменения определяющих емкостей на всем маршруте своего перемещения

$$
\left.\begin{array}{l}
C_{1}=(4-\sim 0.006) \cdot C^{*}=(12.8-\sim 0.02) \\
C_{2}=(\sim 0.006-4) \cdot C^{*}=(\sim 0.02-12.8)
\end{array}\right\} \mathrm{pF} .
$$

Располагая этой информацией и минимальной скоростью перемещения капли $v$ в электрическом поле (2, рис. 1) мы оценили эффективное стартовое значение $q_{S}^{\text {eff }}$, обеспечивающее перемещение капли на любом этапе ее смещения, независимо от динамики перераспределения между емкостями $C_{1}$ и $C_{2}$ :

$$
\left.\begin{array}{l}
q_{1}=\frac{Q}{S_{1}}=\frac{4 C^{*} U_{1}}{S_{1}} \approx 2.4 \cdot 10^{-11} \\
q_{2}=\frac{Q}{S_{2}}=\frac{0.006 \cdot C^{*} U_{2}}{S_{2}} \approx 3.2 \cdot 10^{-8}
\end{array}\right\} q^{\mathrm{eff}} \mathrm{C} / \mathrm{cm}^{2},
$$

где $S_{i}$ - контактные площади капли с соответствующими электродами, $U_{i}-$ падения напряжений на конденсаторах $C_{1}$ и $C_{2}$, обеспечивающие устойчивое перемещение капли.

Емкость электродных участков, не экранированных каплей, не вносит никаких возмущений в представленные выше результаты, поскольку их значения ничтожны и лежат за пределами чувствительности используемых нами приборов. Более того, они подключены параллельно участкам, экранированным водным диэлектриком, что позволяет рассматривать их как параллельно подключенные конденсаторы очень малой емкости. Результирующая емкость такой системы полностью определяется емкостью конденсаторов с жидким диэлектриком.
Управляемая результативность перемещения капли при той же плотности заряда $q^{\text {eff }}$ может быть сохранена и при значительно меньших напряжениях, не превышающих 20-30V [1]. Однако такие напряжения окажутся эффективными лишь при использовании более тонких экранирующих пленок с более высокими значениями диэлектрической проницаемости $\varepsilon$. К их числу относятся оксиды тантала или гафния [14].

Анализ вышепредставленных результатов базируется на эквивалентной схеме двух последовательно соединенных конденсаторах $C_{1}$ и $C_{2}(2)$, которые, как отмечалось выше, сами по себе оказываются сложно структурированными. Поэтому любой из конденсаторов $C_{1}$ и $C_{2}$ можно рассматривать как систему из трех ${ }^{1}$ последовательно соединенных конденсаторов $C_{i j}$ :

$$
\begin{gathered}
C_{1}=\frac{C_{11} \cdot C_{12} \cdot C_{13}}{C_{12} \cdot C_{13}+C_{11} \cdot C_{13}+C_{11} \cdot C_{12}} \approx \frac{C_{11} \cdot C_{13}}{C_{11}+C_{13}}, \\
C_{2}=\frac{C_{21} \cdot C_{22} \cdot C_{23}}{C_{22} \cdot C_{23}+C_{21} \cdot C_{23}+C_{21} \cdot C_{22}} \approx \frac{C_{21} \cdot C_{23}}{C_{21}+C_{23}},
\end{gathered}
$$

в подстрочных символах которых первая цифра $i=1$ или 2 относится к соответствующей контактной зоне рис. 2, $a$, вторая $j-$ к соответствующему конденсатору этой зоны: 1 - к конденсатору с полиэтиленовым диэлектриком, 2 - с тефлоновым и 3 - с $\mathrm{H}_{2} \mathrm{O}$. Если ввести дополнительные обозначения: $Q$ и $U_{i j}-$ величина заряда и падение напряжения на соответствующем конденсаторе; $h_{i j}$ - толщины соответствующих диэлектрических прослоек рассматриваемых здесь конденсаторов $C_{i j}$, то составляющие емкости $C_{i 1}$ и $C_{i 2}$ могут быть представлены очевидными уравнениями

$$
C_{i 1}=\frac{Q}{U_{i 1}}=\frac{\varepsilon_{0} \varepsilon_{i 1} S_{i}}{h_{i 1}}, \quad C_{i 2}=\frac{Q}{U_{i 2}}=\frac{\varepsilon_{0} \varepsilon_{i 2} S_{i}}{h_{i 2}} .
$$

Толщины ${ }^{2}$ диэлектрических слоев $h_{i j}$, как и отношения $\varepsilon_{i j} / h_{i j}$, различаются на порядки. Простые расчеты, основанные на реальных параметрах применяемых нами диэлектриков, показывают, что при симметричном расположении капли на электродных пло- щадках, когда $S_{1}=S_{2}, C_{i 1}: C_{i 2}: C_{i 3}=0.01: 2.90: 0.01 \mathrm{nF}$. Поэтому результирующие емкости конденсаторов каждой из зон (5) определяются наименьшими емкостями $C_{i 1}$ и $C_{i 3}$. Так что толщина и диэлектрические свойства $\mathrm{H}_{2} \mathrm{O}$, как и полиэтиленовой пленки, полностью определяют заряд и кинетику перемещения капли.

Напряжение, падающее на паре управляемых электродов с каплей, перераспределяется между переменными взаимосвязанными конденсаторами

$$
U=\frac{Q}{C}=\underbrace{\frac{Q}{C_{1}}}_{U_{1}}+\underbrace{\frac{Q}{C_{2}}}_{U_{2}}=U_{1}+U_{2} .
$$

\footnotetext{
${ }^{1}$ Реальная емкость „тефлонового“ конденсатора $C_{i 2}$ в сотни раз превышает $C_{i 1}$ и $C_{i 3}$.

${ }^{2}$ Измерение толщин диэлектрических слоев проводилось интерференционным методом на микроинтерферометре МИИ-4. Из-за оптической прозрачности слои экранировались тонкими отражающими $\mathrm{Al}$ пленками, наносимыми методом термовакуумного напыления.
} 


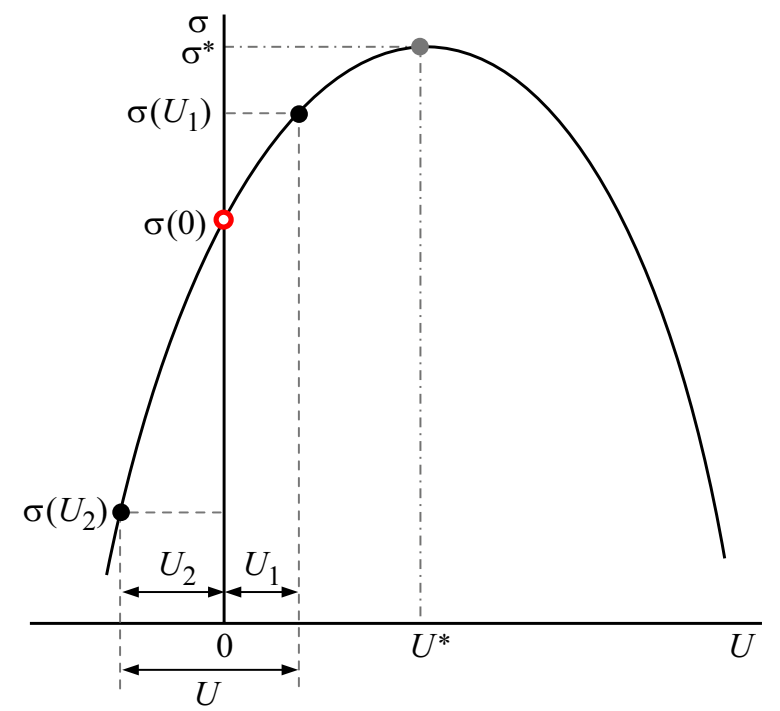

Рис. 3. Графическая иллюстрация уравнения (11). Электрокапиллярная кривая отражает перемещение капли к отрицательному электроду независимо от поляризации токоподводящих электродов.

Отсюда на старте и финише своего перемещения с одной площадки на другую практически все напряжение падает на участки с наименьшей контактной площадью капли с электродом. Именно на этих временных этапах происходит электрический пробой изолирующих пленок, если подаваемое напряжение превышает критическое значение.

На старте своего перемещения капля практически полностью располагается в пределах анодно-поляризованной первой зоны (рис. 2), обеспечивая перераспределение $U=U_{1}+U_{2}$ (7) в пользу катодно-поляризованного фрагмента капли с малой контактной площадью $S_{2}: U_{2} \gg U_{1}$ и $\sigma\left(U_{1}\right) \approx \sigma(0)$ (рис. 3). При равномерном распределении капли на двух площадках $U_{2} \approx U_{1}$ и $\sigma\left(U_{2}\right) \ll \sigma(0) \ll \sigma\left(U_{1}\right)$. На финише перемещения капля завершает свой переход на катодно-поляризованную площадку № 2, полностью экранируя ее и перераспределяя тем самым потенциал в пользу остаточно экранированной анодно-поляризованной зоны № 1: $U_{2} \ll U_{1}$ и $\sigma\left(U_{2}\right) \approx \sigma(0)$.

Для вскрытия механизма управляемого перемещения капли по поверхности с изолированными электродами воспользуемся классическим уравнением Липпмана [7]:

$$
\frac{d \sigma}{d U}=-q_{S}=-\frac{C}{S}\left(U-U^{*}\right),
$$

записанным для контактирующего с подложкой фрагмента капли площадью $S$. Это подразумевает, что поверхностная энергия $\sigma$ соответствует поверхностному натяжению капли $\sigma_{L S}$, контактирующей с твердой матрицей.
Проинтегрируем (8), представив результат в виде уравнений:

$$
\begin{aligned}
& \sigma(U)=-\frac{C}{2 S}\left(U-U^{*}\right)^{2}+A \\
& \sigma(U)=\sigma^{*}-\frac{C}{2 S}\left(U-U^{*}\right)^{2},
\end{aligned}
$$

где $U^{*}$ - потенциал нулевого заряда, соответствующий максимуму электрокапиллярной кривой (8); постоянная интегрирования $A=\sigma^{*}$, поскольку при $U=U^{*}$ межфазное натяжение в экстремальной точке принимает наибольшее значение $\sigma^{*}$, соответствующее точке нулевого заряда.

С другой стороны, при $U=0$

$$
\sigma(0)=\sigma^{*}-\frac{C}{2 S} U^{* 2}, \text { откуда } \sigma^{*}=\sigma(0)+\frac{C}{2 S} U^{* 2} \text {. }
$$

Результат последующей подстановки (10) в (9) определяет характер изменения межфазного натяжения $\sigma$ от приложенного напряжения $U$ :

$$
\sigma(U)=\sigma(0)+\frac{C}{2 S}\left[U^{* 2}-\left(U-U^{*}\right)^{2}\right] .
$$

Графическая иллюстрация этого уравнения представлена на рис. 3, где начало координат электрокапиллярной кривой определяется численным значением $\sigma(0)$ неполяризованной капли.

Особенность проводимых нами экспериментов заключается в том, что контактная площадь $S$, как и прикладываемый потенциал $U(7)$, перераспределяются между зонами рис. 2 в процессе перемещения капли с одной площадки на другую. Следовательно, фрагменты капли, контактирующие с каждым из управляющих электродов, находятся под своими потенциалами, избирательно трансформирующими $\sigma\left( \pm U_{i}\right)$ :

$$
\sigma\left(U_{i}\right)=\sigma(0)+\frac{C_{i}}{2 S_{i}}\left[U^{* 2}-\left(U_{i}-U^{*}\right)^{2}\right] .
$$

Для выяснения места локализации $\sigma(0)$ на электрокапиллярной кривой мы провели серию контрольных опытов с видеорегистрацией скорости перемещения капли при фиксированном $U$. На рис. 4 представлены эти результаты. Они, в сочетании с другими контролируемыми параметрам, указывают на принадлежность стартового значения $\sigma(0)$ к восходящей ветви электрокапиллярной кривой. Действительно, для всего диапазона используемых нами $U=100-300 \mathrm{~V}$ поверхностная энергия катодного участка $\sigma\left(U_{2}\right)$ всегда меньше анодного $\sigma\left(U_{1}\right)$, что обеспечивает вытеснение капли лишь к отрицательному электроду, а увеличение скорости перемещения капли с ростом $U\left(U<U^{*}\right)$ свидетельствует о возрастающей разнице $\Delta \sigma_{i}$ активных зон перемещающейся капли. Однако при постоянном $U$, несмотря на экстремальное поведение результирующей емкости (1, рис. 1$)$, процесс 


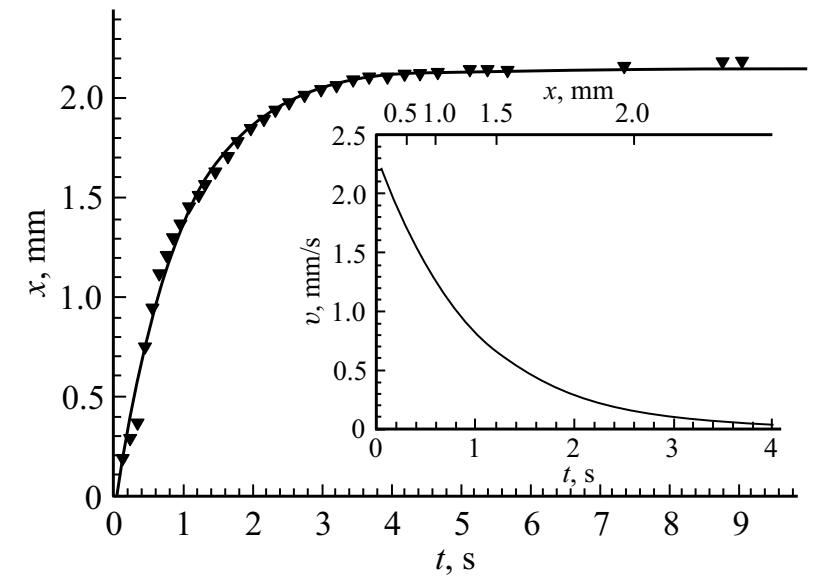

Рис. 4. Перемещение капли во времени при $U=100 \mathrm{~V}$, отслеживаемое по переднему фронту. На вставке: изменение скорости перемещения капли $v$ от времени $t$ при $U=100 \mathrm{~V}$.

перемещения капли характеризуется только монотонным снижением $v$, что указывает (вставка рис. 4) на возрастающую кривизну восходящей ветви $\sigma(U)-$ кривой рис. 3, уменьшающую эффективность воздействующего параметра $\Delta \sigma=\sigma\left(U_{1}\right)-\sigma\left(U_{2}\right)$. Этим условиям удовлетворяет $U<U^{*}$. В противном случае в зависимости от кривизны и соответствующих изменений $\Delta \sigma_{i}$, может меняться не только скорость перемещения капли, но и ее направление. Таким образом, координаты поверхностной энергии капли $\sigma(0)$ на границе с подложкой, как и последующие изменения $\sigma\left(U_{i}\right)$, сопряжены с восходящей ветвью электрокапиллярной кривой, как это и изображено на рис. 3.

В пределах используемых нами значений $U$ изменение полярности подключения электродов или их заземления не сказывается на направленности перемещения капли, поскольку приоритет смачиваемости остается за отрицательным электродом, на который и стремится переместиться капля. Все это убедительно подтверждается контрольными опытами, в которых источником питания служила предварительно заряженная до 100-300 V батарея конденсаторов большой емкости. Это свидетельствует, что все фиксируемые нами процессы контролируются восходящей ветвью электрокапиллярной кривой.

Различная смачиваемость подложки анодным и катодным фрагментами капли неминуемо приводит и к различию краевых углов смачивания. Действительно, краевой угол смачивания $\theta$ неполяризованного $\left(\sigma_{L S} \equiv \sigma(0)\right)$ электрода подчиняется классическому уравнению Юнга [15]:

$\sigma_{L S}=\sigma_{G S}-\left.\sigma_{G L} \cdot \cos \theta\right|_{U=0} \quad$ или $\left.\cos \theta\right|_{U=0}=\frac{\sigma_{G S}-\sigma_{L S}}{\sigma_{G L}}$,

$\sigma_{L S}=\sigma_{G S}-\left.\sigma_{G L} \cdot \cos \theta\right|_{U=0} \quad$ или $\left.\cos \theta\right|_{U=0}=\frac{\sigma_{G S}-\sigma_{G L}}{\sigma_{L S}}$,

в котором подстрочный символ $L$ относится к жидкости, $S$ - к твердой подложке и $G-$ к газу.
При приложении внешнего потенциала поверхностная энергия $\sigma_{L S}$ каждой зоны испытывает значительные возмущения, в то время как $\sigma_{G S}$ и $\sigma_{G L}$, согласно [8], остаются неизменными. Независимость этих параметров от внешнего напряжения мы подтвердили опытами с индуцированными зарядами, в которых капля располагалась лишь на одном катодно- или анодно-поляризованном $(0-15 \mathrm{kV})$ электроде без подключения второго. Действительно, капля не реагировала на подключение и отключение внешнего источника, независимо от того, находилась ли она на неэкранированном металлическом электроде или на электроде, экранированном диэлектрическими пленками. Это позволяет утверждать, что при неизменности $\sigma_{G S}$ и $\sigma_{G L}$ численное значение краевого угла смачивания $\sigma_{L S} \equiv \sigma\left(U_{i}\right)$, поляризуемого фрагмента капли, определяется уравнением

$$
\left.\cos \theta\right|_{U_{i}}=\frac{\sigma_{G S}-\sigma\left(U_{i}\right)}{\sigma_{G L}},
$$

которое с учетом (12), устанавливает корректирующее влияние электрического поля на краевые углы смачивания рассматриваемых фрагментов капли

$$
\left.\cos \theta_{i}\right|_{U_{i}}=\frac{\sigma_{G S}-\sigma(0)}{\sigma_{G L}}-\frac{C_{i}}{2 \sigma_{G L} S_{i}}\left[U^{* 2}-\left(U_{i}-U^{*}\right)^{2}\right] .
$$

Здесь $C_{i}$ соответствует определяющим емкостям составных конденсаторов $C_{1}$ и $C_{2}(5)$, численные значения которых

$$
C_{i}=\frac{C_{i 3}}{1+\frac{C_{i 3} h_{i 1}}{\varepsilon_{0} \varepsilon_{i 1} S_{i}}},
$$

практически полностью исключают заметную роль тефлоновой прослойки $\left(h_{i 2}, \varepsilon_{i 2}\right)$. Это позволяет переписать (15) в виде

Как отмечалось выше (7), перемещение капли с одного электрода на другой перераспределяет $U_{i}$ между электродами. При доминировании $U_{i}$ над $U^{*}$ численным значением $U^{*}$ можно пренебречь, что позволяет представить (17) в более упрощенном виде

$$
\begin{aligned}
\left.\cos \theta_{i}\right|_{U_{i}}=\left.\cos \theta_{i}\right|_{U=0} & -\frac{C_{i 3}}{2 \sigma_{G L}\left(S_{i}+\frac{C_{i 3} h_{i 1}}{\varepsilon_{0} \varepsilon_{i 1}}\right)} \\
& \times\left[U^{* 2}-\left(U_{i}-U^{*}\right)^{2}\right] .
\end{aligned}
$$

согласующимся с [8].

Но процесс перемещения капли сопровождается синхронным изменением $U_{i}$ (рис. 3) на сопряженных площадках. Поэтому на старте своего перемещения краевой угол смачивания набегающего (катодного) участка капли описывается уравнением (18) с предельными значениями $U_{i} \lesssim U$, а анодно-поляризованного сбегающего фрагмента капли - уравнением (17) с предельно низкими значениями $U_{i} \gtrsim 0$. Завершающий этап перемещения капли на катодную площадку должен характеризоваться 

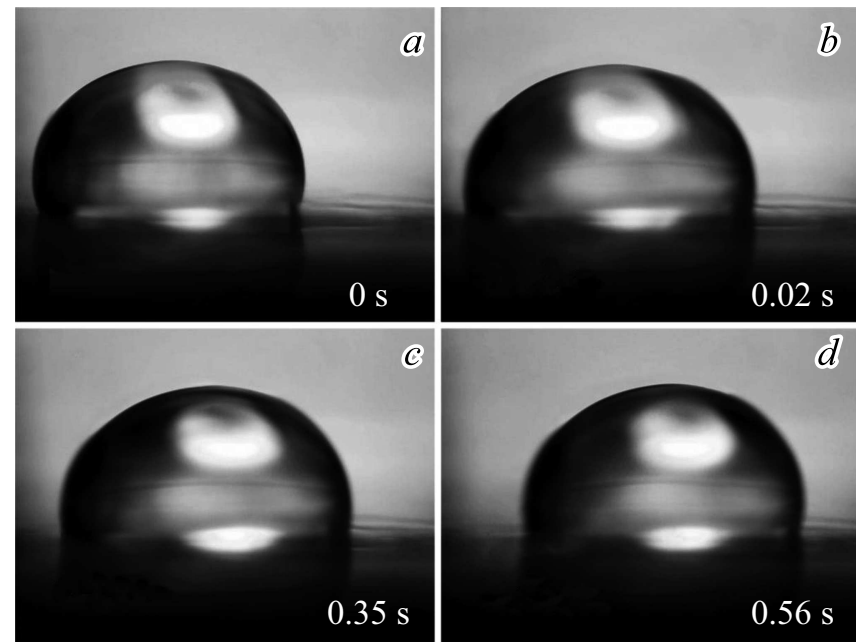

Pис. 5. Динамика краевых углов смачивания активных зон при перемещении капли с одного электрода на другой. $U=100 \mathrm{~V}$. $a-$ стартовое положение капли; $b-d-$ положение капли в различные моменты времени.

зеркальным изменением $U_{i}$ и краевых углов смачивания относительно стартовых значений. В результате $\theta$ переднего фронта капли на конечном этапе перемещения должно описываться уравнением (17) с малыми значениями $U_{i} \gtrsim 0$, а сбегающего анодно-поляризованного участка - уравнением (18) с наибольшим значением управляющего напряжения $U_{i}$, приближающимся к $U$.

Раскадровка видеосъемки подтверждает синхронные изменения $\theta_{i}$ активных зон мигрирующей капли (рис. 5). Однако количественное несоответствие экспериментально найденных краевых углов смачивания и рассчитанных по уравнениям (17) и (18) указывают на неравновесность (динамичность) зафиксированных значений $\theta_{i}$, наиболее ярко выраженных на сбегающем фрагменте капли. Действительно, экспериментально найденное значение краевого угла смачивания $\theta_{1} \approx 94^{\circ}$ тыльного фрагмента движущейся капли (рис. $5, b$ ) в момент начала перемещения оказалось на $\sim 10-12 \%$ меньше расчетного (17). Причину этого мы видим в динамике силового воздействии переднего фронта на геометрию увлекаемой им капли. Действительно, на старте поляризации происходит перезарядка двойного электрического слоя с постепенным наращиванием $\Delta \sigma=\sigma_{1}-\sigma_{2}$ до некоторого критического значения, обеспечивающего перемещение капли. До этого момента капля, оставаясь на месте, деформируется, накапливая для перемещения энергию. Эта деформация должна осуществляться за счет переноса массы жидкости в пограничном слое с участка с меньшим значением $\sigma$, следовательно, и $\theta$, к участку с большими значениями этих параметров. Когда $\Delta \sigma$ превышает критическое значение, накопленная энергия трансформируется в кинетическую, что подтверждается фактором „мгновенного“ перемещения капли на некоторое расстояние (рис. $5, b$ ), обеспеченное накопленной энергией. Но обновление контактной площадки при каждом таком переходе требует подзарядки двойного электрического слоя межфазной границы каждого из электродов при снижающейся эффективности воздействующего параметра $\Delta \sigma$ (рис. 3). Это должно определять прерывистый механизм перемещения капли на всем этапе поляризации с различными временами оседлости, зависящими от параметров опыта и площади ее соприкосновения со вторым электродом. Все это подтверждается результатами скоростной (240 fps) видеосъемки, согласно которым импульсный переход завершается практически полной остановкой капли в контактной области с электродом при продолжающемся смещении основной ее массы без значительного изменения координат обновленной контактной площади. Инерционность рассматриваемой системы перебрасывает поток жидкости на передний фронт капли, увеличивая тем самым краевой угол смачивания на набегающем ее фронте и уменьшая на сбегающем. Это, в свою очередь, указывает на доминирующую роль циркулирующих потоков жидкости вдоль пограничного слоя, определяющих механизм перемещения капли при различающихся $\sigma$ на фронтальной и тыльной сторонах капли. Наглядным подтверждением этого служит завершающий этап перемещения капли на второй электрод, включая ее остановку. Продолжающаяся поляризация неподвижной капли на тех же электродах уменьшает ее высоту и увеличивает массу жидкости в анодном ее фрагменте, растягивая жидкость в направлении анода. Краевой угол смачивания анодного участка при этом уменьшается на $3-4^{\circ}$, а координаты переднего фронта, как и его $\theta$, остаются неизменными. Активная переброска граничащей с матрицей жидкости методом „пробуксовки“ поверхностного слоя прекращается при отключении напряжения. Капля принимает стандартную форму с присущим ей значением $\theta$ для неполяризованной капли. Все эти процессы повторяются при каждой последующей поляризации. Эти наблюдения позволяют утверждать, что именно этот поток меняет геометрию тыльного фрагмента капли, принуждая краевой угол смачивания сбегающего участка (рис. 2 и $5, b$ ) уменьшаться за счет перебрасываемого потока жидкости по межфазной границе $L S$.

Таким образом, представленные здесь результаты вскрывают природу передвижения капли по экранированным электродным площадкам.

\section{Список литературы}

[1] Kwon C.S., Moon H., Kim Ch.-J. // J. Microelectromech. Syst. 2003. Vol. 12. N 1. P. 70-80.

[2] Schultz P., Cumby B., Heikenfeld J. // Appl. Opt. 2012. Vol. 51. N 17. P. 3744-3754.

[3] Kilaru M.K., Cumby B., Heikenfeld J. // Appl. Phys. Lett. 2009. N 94. P. 041108. 
[4] Preston D.J., Anders A., Barabadi B., Tio E., Zhu Y., Dai D.A., Wang E.N. // Appl. Phys. Lett. 2016. N 109. P. 244102.

[5] Mishra K., Ende van den D., Mugele F. // Micromachines. 2016. Vol. 7. N 102. P. 1-24.

[6] Heikenfeld J., Steckl A.J. // Appl. Phys. Lett. 2005. Vol. 86. P. 011105.

[7] Герасимов Я.И. Курс физической химии. В 2 т. Том 2. М.: Химия, 1973. $624 \mathrm{c.}$

[8] Mugele F., Baret J.-Ch. // J. Phys.: Condens. Matter. 2005. N 17. R. 705-774.

[9] Ren H., Fair R.B., Pollack M.G., Shaughnessy E.J. // Sens. Actuator. B. 2002. Vol. 87. P. 201-206.

[10] Cho S.K., Moon H., Kim C.-J. // J. Microelectromech. Syst. 2003. Vol. 12. N 1. P. 70-80.

[11] Fan S-K., Yang H., Wang T.-T., Hsu W. // Lab Chip. 2007. Vol. 7. P. $1330-1335$.

[12] Cooney C.G., Chen C.-Y., Emerling M.R., Nadim A., Sterling J.D. // Microfluid. Nanofluid. 2006. Vol. 2. N 5. P. 435-446.

[13] Torkkeli A. Droplet microfluidics on planar surface. Espoo: Otamedia Oy., 2003. 194 p.

[14] Guha I.F., Kedzierski J., Abedian B. // Appl. Phys. Lett. 2011. Vol. 99. P. 024105.

[15] Сумм Б.Д., Горюнов Ю.В. Физико-химические основы смачивания и растекания. М.: Химия, 1976. 232 с. 